\title{
BMJ Open Treatable but not curable cancer in England: a retrospective cohort study using cancer registry data and linked data sets
}

\author{
Rachel White, ${ }^{1}$ Fintan Stanley (D) , ${ }^{1,2}$ Jen Than, ${ }^{1,2}$ Archie Macnair, ${ }^{1,3}$ \\ Joanna Pethick, ${ }^{2}$ Gregory Fallica, ${ }^{1}$ Luke Hounsome, ${ }^{2}$ Jane Maher ${ }^{1,4}$
}

To cite: White R, Stanley F, Than J, et al. Treatable but not curable cancer in England: a retrospective cohort study using cancer registry data and linked data sets. BMJ Open 2021;11:e040808. doi:10.1136/ bmjopen-2020-040808

- Prepublication history and additional material for this paper are available online. To view these files, please visit the journal online (http://dx.doi org/10.1136/bmjopen-2020040808).

RW and FS are joint first authors.

Received 22 May 2020 Revised 03 December 2020 Accepted 07 December 2020

Check for updates

(c) Author(s) (or their employer(s)) 2021. Re-use permitted under CC BY-NC. No commercial re-use. See rights and permissions. Published by BMJ.

${ }^{1}$ Macmillan Cancer Support, London, UK

${ }^{2}$ National Disease Registration Serivce, Public Health England, London, UK

${ }^{3}$ Royal College of Radiologists, London, UK

${ }^{4}$ Mount Vernon Cancer Centre, Northwood, UK

Correspondence to

Rachel White;

rwhite@macmillan.org.uk

\section{ABSTRACT}

Objectives This study estimates the prevalence of cancers that are categorised as treatable but not curable (TbnC) in England. It provides a quantification of the population and a framework to aid identification of this group to enable the design of tailored support services. Design Through consultation with clinical and data experts an algorithmic definition of TbnC was developed. Using cancer registry data sets, with five other linked data sets held by the National Disease Registration Service, the algorithm was applied as part of this retrospective cohort study to estimate the size and characteristics of the TbnC population.

Setting and participants The health data records of 1.6 million people living with cancer in England in 2015, following a cancer diagnosis between 2001 and 2015, were retrospectively assessed for TbnC status.

Results An estimated 110615 people in England were living with TbnC cancer at the end of 2015, following identification of TbnC cancer between 2012 and 2015. In addition, 51946 people fit the initial search criteria but were found to have been in their last year of life at the end of 2015 and therefore considered separately here as end of life cases. A further 57117 people in England were initially identified as being at high risk of recurrence or having their life being shortened by cancer but did not fit the TbnC conceptual framework and were excluded, but their results are also reported under 'group B'.

Conclusions A population living with TbnC cancer can be identified using data currently collected on a national scale in England. This large population living with TbnC cancer requires personalised treatment and support.

\section{INTRODUCTION}

There is an important subgroup of people with cancer who, although ultimately their cancer is very rarely cured (eradicated completely), have the option to receive treatments that can slow the progression of their cancer, prolong life and control symptoms. ${ }^{1}$ This group of people can be referred to as living with 'treatable but not curable' (TbnC) cancer. Here we aim to use English cancer
Strengths and limitations of this study

- The algorithm used to define treatable but not curable (TbnC) status was developed through repeated consultation with healthcare professionals. This strengthened the work by validating the relevance of the concept to clinical practice and by developing consensus among those caring for people living with cancer.

- However, reliance on clinical opinions can increase the subjectivity and inconsistency within the criteria and it is likely to require updating as new treatment and diagnostic processes emerge.

- The results are produced from algorithmic analysis of routinely collected health data from multiple linked data sets. This offers a large and high-quality evidence base, covering all England, and enables further use of this data in guiding care and policy.

- Conversely, reliance of the algorithmic definition on the cancer registry and linked data sets means that the analysis is limited to a time frame were the data sets were of sufficient quality and completeness.

- TbnC is a heterogeneous group and marginal cases can challenge any definition however multiple criteria were used to reduce potential errors.

registry and linked data sets to quantify the population living with TbnC cancer.

People living with TbnC cancer are a heterogeneous group with different survival lengths and a variety of treatment options. ${ }^{1}$ In our terminology a patient's TbnC status does not always mean being in active treatment, nor does it rule out that treatments received would include those described by clinicians as 'radical' or 'curative' . Rather, not curable refers to the expectation that the cancer is highly unlikely to be eradicated and there is a high chance that, in the absence of other more imminent causes of death, this cancer will lead to death. Most people in the TbnC group will be living with a cancer that is not curable from diagnosis until their death. This 
means treatment decisions need to be taken in the context of higher levels of uncertainty in their outcome and more 'safety net' support should be planned for these patients; these might include cancer-specific information, psychological support, exercise and energy advice or financial and work support.

TbnC is not a widely used terminology, however, no alternative with a clear consistent definition exists. Related concepts like advanced, progressive, chronic, palliative, terminal, incurable or life-limiting cancer do not have a standard definition that applies to all cancer types and settings. They also come with preconceptions that can build barriers with people living with cancer rather than opening up conversations for shared decision-making. We acknowledge the overlap in these terms and seek to clarify the language and focus of such research by using the single transparent TbnC terminology.

People living with TbnC cancer go through various diagnostic and treatment pathways but they often face the shared experience of uncertainty around the progression of their cancer. Living with TbnC cancer can be a long and complex experience, potentially under the care of several healthcare professionals and undergoing multiple treatments. ${ }^{1}$ For instance, research on people living with myeloma showed that the key issue they reported after their diagnosis and while not in active treatment, was uncertainty about their future. ${ }^{2}$ Other studies have shown that people with advanced colorectal cancer mention continual uncertainty about future functional decline and prognosis. ${ }^{3}$ Uncertainties often relate to the timescale and trajectory of the disease and the patient's perceived lack of control or ability to make plans. This can mean that people feel as if they are 'living from scan to scan'. The impact of this uncertainty extends beyond prognosis to broader areas of life, such as planning for the future, providing for family, employment, finances and retirement. ${ }^{5}$ People living with TbnC cancer can have a range of medical or physical needs, such as pain and fatigue, as well as more cancer specific or cancer treatment-related symptoms. ${ }^{2}$ Some people with TbnC cancer even feel in good health for periods, reporting that they need 'to get over the shock of being told you have an incurable cancer even though you feel well'. ${ }^{12}$

Previous analysis of a similar population estimated the number of people with 'progressive' cancer across four cancer types. ${ }^{6}$ This estimate was based on people with metastatic disease, but not in their last year of life. That study concluded that progressive cancer is one of the most resource-dependent phases of the care pathway, both in terms of expense and need for support. Subsequently, the Three Cancer Groups framework identified a group of cancers with 'intermediate survival' (typically more than a year but less than $80 \%$ 5-year survival), for which cancer is a complex ongoing disease. ${ }^{7}$ This work called for shared care between patients and clinicians to preserve quality of life through a balance of acute interventions, chronic illness management, palliative care principles, acknowledgement that cancer is likely to be life-limiting and recognition of people's move to the dying phase.

There is a point, in the cancer journey, when people who have lived with TbnC cancer are more helpfully described as 'approaching the end of life' (EoL). There is no clear distinction on when this happens, but other analyses have used the definition of the last year of life, ${ }^{8}$ which is the approach we have taken here. However, it has to be noted that this definition may not reflect typical access to EoL services. ${ }^{9}$

Some cancers that are defined as TbnC in this paper may not be recognised as such by the person living with cancer or healthcare professionals. This can be due to difficulties in predicting an individual's prognosis based on their personal and disease characteristics, differences in how clinicians, people living with cancer and others think about terms like 'not curable' and a possible gap between the hoped outcome for their condition and impartial outlooks. In this study, we aim to take a datadriven approach to TbnC, rather than focusing on people who would self-identify as TbnC or people who would be identified as TbnC by their medical team. This has the advantage of not being influenced by person to person variations in the definition of TbnC but, inevitably, it will mean that, for some people identified in this group, the person living with cancer or their medical team would disagree with the TbnC classification.

Our objective in this study was to quantify the number of people living with TbnC cancer in England. This required us to refine the concept of TbnC cancer into an algorithmic definition using the available variables in the cancer registry and linked databases. Sizing the TbnC population will build credibility to the TbnC concept, demonstrate its importance to policymakers and the general public and aid in the design of services that can provide personalised care to meet the individual concerns of people within this population. The algorithmic definition will add precision and standardisation to the definition and allow further research analysis into the characteristics and needs of the population.

\section{METHODS}

\section{Definition development}

While this study focuses on the quantification of TbnC cases in England, earlier work was done to establish a working definition for 'TbnC' cancer (figure 1). Briefly, previous unpublished work that informed TbnC work included qualitative research on advanced care planning, which involved interviews with people living with cancer $(n=13)$ and health and social care providers $(n=30)$. The actual term 'TbnC' was then developed and qualitative interviews with people living with TbnC cancer $(n=8)$ were carried out to understand how they describe and define living with their diagnosis. The definition was then tested and refined through interviews with stakeholders and experts in the field, including palliative care consultants, general practitioners (GPs), cancer specialist 


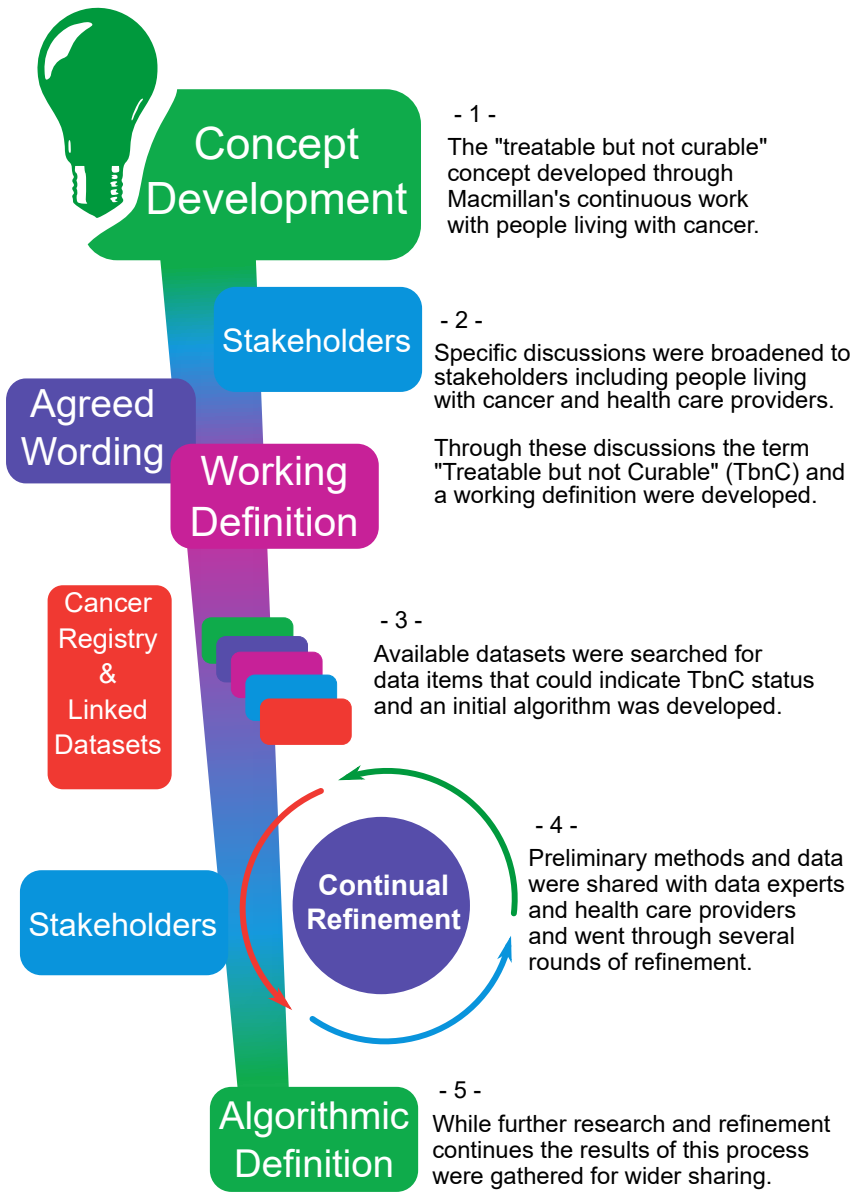

Figure 1 Concept development flow diagram; a summary of the path from concept development to the TbnC algorithm and results of this analysis. TbnC, treatable but not curable

nurses, haematology nurses and physiotherapists, specifically this involved multi-site structured interviews with healthcare providers $(n=21)$ across the UK. Additional in-depth structured interviews $(n=11)$ with people living with TbnC cancer were also carried out. The term TbnC and their populations specific needs were further tested through thematic analysis of online cancer forum threads, a narrative literature review of published research on TbnC cancer (including searches on other related terms) and secondary consolidation and thematic analysis of the results of all the aforementioned work.

The actual term 'Treatable but not Curable' was then settled on to describe this cancer and the experience, with the intent that it is meaningful, acceptable to people living with cancer and professionals and useful in practice. A working definition of TbnC was also developed-the result: 'TbnC cancer refers to people living with cancer that can very rarely be cured (eradicated completely) but can be treated to slow the progression of their cancer, prolong life and control symptoms'.

\section{Algorithm development}

Next, we began the process of counting people living with TbnC cancer in England. To do this, we took a retrospective cohort approach, using the National Cancer Registration
Data set and a selection of linked databases. ${ }^{10}$ Namely, we used the linked data from the systematic anti-cancer therapy (SACT) data set, radiotherapy data set (RTDS), National Cancer Waiting Times Monitoring data set and Hospital Episodes Statistics (admitted and outpatient) dataset; the details of these data sets and their linkage are available from the National Disease Registration Service (NDRS). ${ }^{11}$ No single field in these data sets would allow direct identification of TbnC cancer, therefore we constructed a set of search criteria to define this group algorithmically using various fields across multiple data sets. Although we recognised that each criterion provides only partial information in isolation, by combining them, we sought to identify the majority of the TbnC cancer group. We took a conservative approach seeking to identify the TbnC cohort using the definitions around which we received the most confidence and consensus.

A preliminary algorithm was developed based on concepts explored in the qualitative description of this group and in the Three Cancer Groups framework. ${ }^{7}$ This included selecting people based on specific cancer types, stages of disease, treatment options or treatment intent. For example, selecting people who received radiotherapy recorded in RTDS as having 'palliative' intent. This initial algorithm was applied to the cancer registry data sets to estimate the number of people meeting each criterion. The methodology and preliminary results of this process were then scrutinised and refined in a workshop involving 25 collaborators, including oncologists, haematologists, researchers, data experts and specialist cancer nurses (figure 2).

A second, more comprehensive, set of search criteria were then used to redevelop the algorithm, which again was used to quantify people living with TbnC cancer. This iteration was informed by a survey of clinical advisors on SACT treatments (criteria 5) and refining the cancer types and stage combo (criteria 1) used to define TbnC status. Alongside the quantification, we carried out an analysis of people identified through more than one search criteria to understand their overlap. Additional consideration was placed on the subset that only met one search criteria to ensure that these were true cases of TbnC cancer, as they could represent margins of the definition, lacking the validation provided when multiple criteria are met.

Our preliminary findings were then reviewed in a second workshop. At this point, the need to differentiate a related cohort ('group B') started to emerge. This group focuses on people with cancers who are not in stage 4 but where the 5 -year net survival rate tends to be $50 \%$ or less. Group B includes people with apparent locoregional solid cancers who may receive aggressive radical treatments but are at high risk of recurrence or having their life being shortened by cancer. It also includes heterogeneous cancer types, such as brain and nonHodgkin's lymphoma, where the chance of being not curable depends on the specific cancer subtype. There is a case for saying that people in group $\mathrm{B}$, like the TbnC group, need support to prepare for EoL, even if they have 


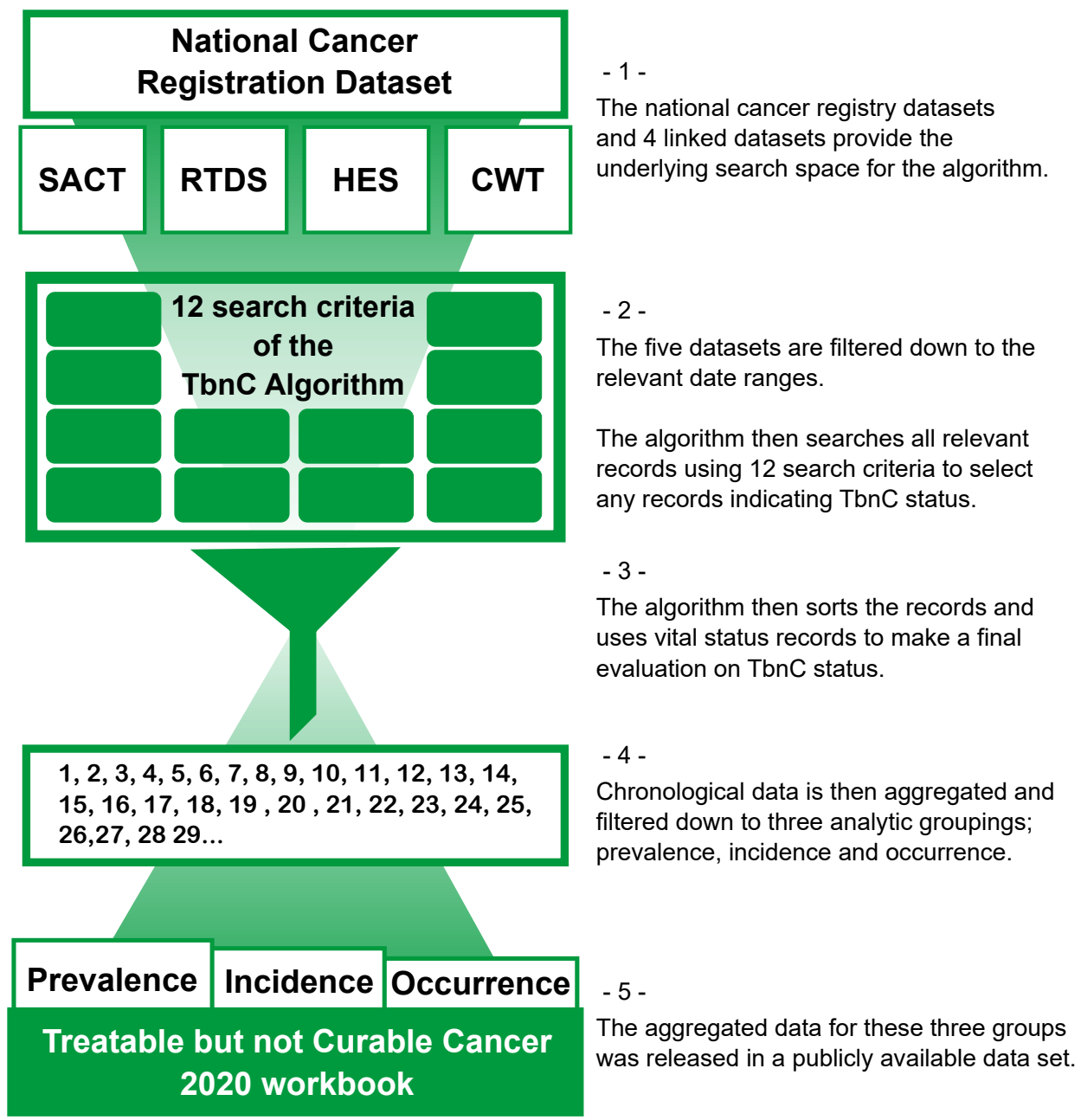

Figure 2 Data flow diagram summarising the steps undertaken during a run of the algorithm used to quantify TbnC cases. CWT,National Cancer Waiting Times Monitoring;HES, Hospital Episodes Statistics; RTDS, radiotherapydata set;SACT, systematic anti-cancertherapy;TbnC, treatable but not curable.

a reasonable chance of receiving successful radical treatment. As such we differentiate the 'TbnC' population from the related population ('group B') but continue to present data for both groups separately in our analysis.

A final iteration of the algorithm was then developed; this included a refinement of the criteria based on Hospital Episode Statistics (detailed protocol available in online supplemental appendix 1). People with TbnC cancer are defined as anyone who met at least one of the final search criteria (online supplemental appendix 2). Two selection criteria of the algorithm can denominate group B cases, but where any of the 12 search criteria indicate TbnC status this was given preference. Another list of criteria was evaluated and discarded from the analysis, as they were determined not to be successful in identifying people living with TbnC cancer (online supplemental appendix 2).

The final 12 search criteria were algorithmically applied to the NDRS data sets to compile a list of times the search criteria are met for each relevant person (figure 2). Each time a search criterion is met by a person, there is an associated date which is used to identify the first time each criterion is met by an individual. This timeline is used within the algorithm to select the earliest TbnC designating event. The person and cancer characteristics in the results data are based on the status at the time of the earliest TbnC designating event.

The TbnC prevalence group includes all those alive at the end of 2015 who were not at EoL (in their last year of life). The algorithm does initially identify the combined TbnC and EoL population, but those at EoL are ultimately excluded by removing people later found to have who died in 2016. Those who are excluded due to being at EoL are presented separately here for completeness and to aid interpretation of the search criteria. A welch two sample t-test was used to test the significance of the difference in mean number of criteria met between TbnC and EoL groups. The t-test was carried out on ln transformed data to normalise the data distribution and medians and ranges are quoted in text for interpretability.

Detailed methods, descriptions of the algorithm's 12 search criteria and data set descriptions are additionally set out in the publicly available workbook and online supplemental appendix 1 . The workbook also contains 
the aggregated data results of the study: http://www.ncin. org.uk/view? rid=4153.

\section{Reporting and data access}

Strengthening the Reporting of Observational studies in Epidemiology (STROBE) cohort reporting guidelines were used. ${ }^{12}$ The NDRS is part of PHE (Public Health England). PHE has been granted specific legal permission to collect information about patients with cancer for specific purposes, without the need to seek consent. These purposes include health improvement and service provision. This permission was granted to PHE through section 251 of the NHS Act $2006 .{ }^{13}$ This support is reviewed annually by the Confidentiality Advisory Group of the Health Research Authority. PHE releases are subjected to strict confidentiality provisions in line with the requirements of the Common Law Duty of Confidentiality, ${ }^{14}$ the General Data Protection Regulation (EU) 2016/679 and the seven Caldicott principles. ${ }^{14-16}$

\section{Patient and public involvement}

Patients were involved in early development, particularly around the 'TbnC' terminology where a patient stakeholder groups were consulted on the applicability of this term. However, it was not possible to involve patients in other later areas of the study design due to data protection restrictions and the technical methods required to do a data linkage analysis.

\section{RESULTS}

Results are presented for the following populations:

\begin{tabular}{|c|c|}
\hline Prevalence & $\begin{array}{l}\text { Estimated number of people living with treatable } \\
\text { but not curable (TbnC) cancer at the end of } 2015 \text {. } \\
\text { Includes those alive at end of } 2015 \text { and having } \\
\text { met criteria between } 2012 \text { and } 2015 \text {. This is a } \\
\text { person's level count, so people are counted once } \\
\text { under the first of the } 12 \text { criteria that they met in } \\
\text { the analysis period. }\end{array}$ \\
\hline Occurrence & $\begin{array}{l}\text { Estimated number of times a distinct TbnC } \\
\text { criterion was met. Includes those alive at end } \\
\text { of } 2015 \text { and having met criteria between } 2012 \\
\text { and } 2015 \text {. This is an event level count, so people } \\
\text { are counted up to } 12 \text { times, once per each new } \\
\text { criteria met. Those who meet the same criteria } \\
\text { more than once are only counted once. }\end{array}$ \\
\hline Incidence & $\begin{array}{l}\text { Estimated number of people who became TbnC } \\
\text { during } 2015 \text {. Includes those meeting criteria in } \\
2015 \text { and not previously during 2012-2014. This } \\
\text { is a person's level count, so people are counted } \\
\text { once under the first of the } 12 \text { criteria that they } \\
\text { met in the analysis period. Vital status is not } \\
\text { considered. }\end{array}$ \\
\hline
\end{tabular}

All three cohorts are additionally split by whether the algorithm indicated 'TbnC' or 'group B' status. Prevalence and occurrence counts are also split by the EoL status of the patients. EoL positive indicates they died within a year of the end of the study period (31 December 2015), that is, at the end of 2015, they were in their last year of life, rather than TbnC.

\section{Prevalence of TbnC cancer in England in 2015}

There were 110615 people in England living with TbnC cancer at the end of 2015 (figure 3A). This counts people based on their first-identified TbnC cancer and includes 12209 people living with breast cancer (11\%), 20432 with prostate cancer (18\%) and 26943 living with haematological cancers $(24 \%)$. There were also a substantial number of colorectal (10\%), lung (7\%) and head and neck cancers $(6 \%)$ (figure 3B). Breaking down TbnC population by stage at diagnosis, stage 4 is most common (43\%).

We additionally report a further 45942 people in England in group B (figure 3A). Group B includes those identified as being at high risk of recurrence or having their life being shortened by cancer but who did not fit the TbnC framework following categorisation. As expected, (criteria 1 will designate most stage 4 cases to TbnC) there are notably less people diagnosed at stage 4 in Group B (7\%), than in TbnC (figure 3C), more group B cases are staged as 'unknown' at diagnosis (43\%).

We consider the TbnC group to exclude people at EoL (in their last year of life). A total of 51946 people fit the initial search criteria but were found by subsequent steps in the algorithm to have been at EoL rather than TbnC. An added 11175 potential group B cases were found to be EoL, in total the algorithm identified 63121 EoL cases (figure 3A). The total prevalence of all people living with a TbnC cancer, a group B cancer or at EoL, was 219678.

\section{Timing of TbnC status at the patient level}

In the prevalence population (people living with TbnC cancer in England at the end of 2015), the median age at time of their cancer diagnosis was 67 and the median age at the earliest TbnC designating event was 68 years (figure 4A) The age distributions at each of these points are broadly similar. Four in 10 were of working age $(40 \%$ aged 15-65) at the time of meeting the TbnC criteria. The male sub-population is skewed towards older ages.

Two-thirds of the people identified had their earliest TbnC designating event within a month of cancer diagnosis but, for some people, there was a gap between their diagnosis and earliest TbnC event. For 16\%, there was a gap of a year or more between diagnosis and earliest TbnC event. The occurrence figures, which count not just the first criteria but also each subsequent one, show that about half (48\%) of all TbnC events happened no more than a month from diagnosis. One quarter occurred after this but within a year, and the final quarter occurred more than 1 year after diagnosis, with $6 \%$ happening more than 5 years after diagnosis (figure 4B).

Looking at specific cancer types, 38\% of people living with TbnC breast cancer and $42 \%$ of people with TbnC skin cancer had a gap of 1 year or more between their initial cancer diagnosis and their earliest TbnC designating event. For $8 \%$ of TbnC breast cancer cases, there is a decade or more between the first cancer diagnosis and 


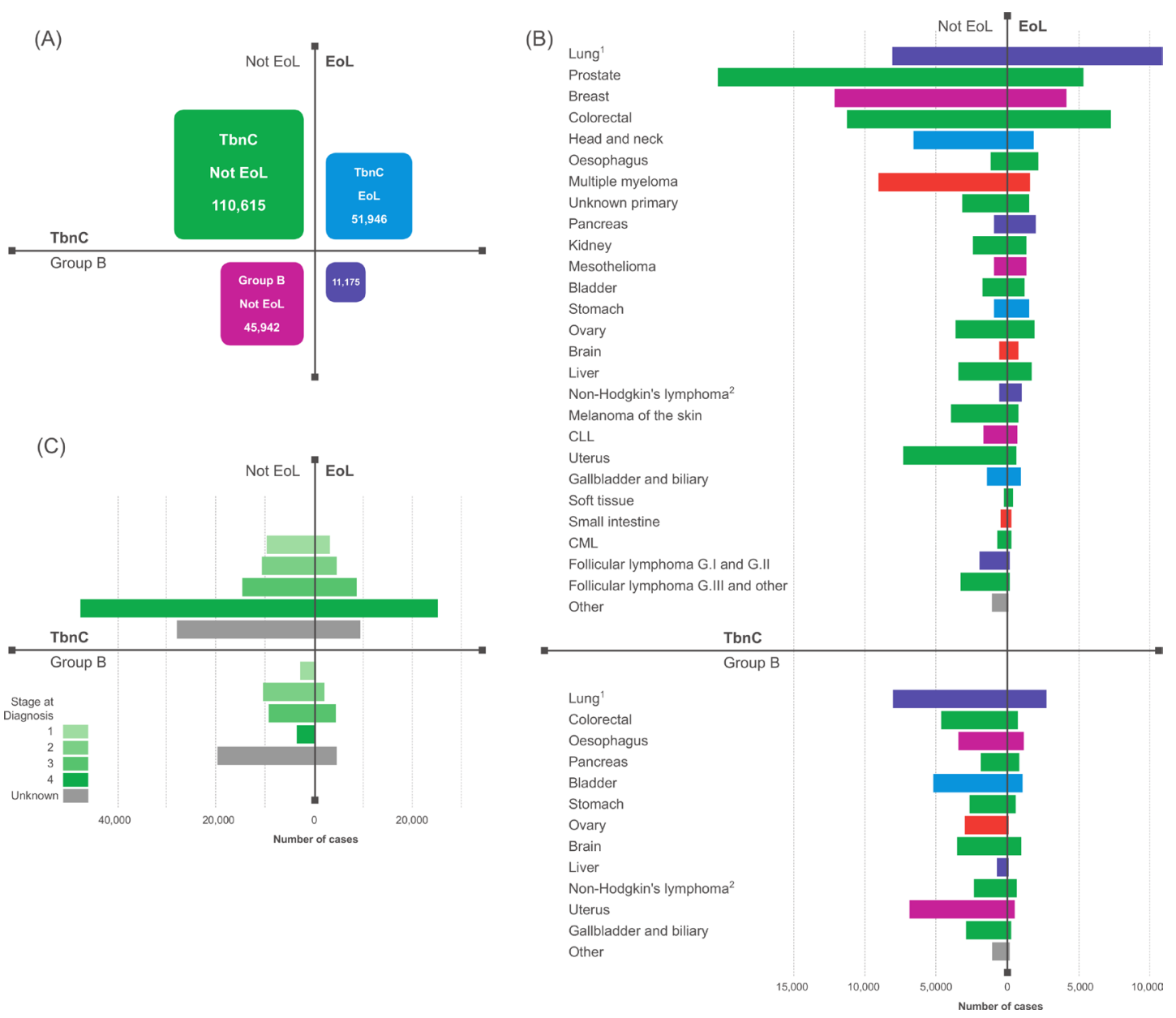

Figure 32015 Prevalence of TbnC cancer in England. (A) Prevalence number of TbnC cancer in England in 2015, 110,615 people living with TbnC cancer and not in their last year of life (TbnC, not EoL), with an additional 51946 in their last year of life (TbnC, EoL). There were also 45952 and 11175 people in group B who were not and were in their last year of life, respectively (group B, not EoL; group B, EoL). (B) Cancer type breakdown of the TbnC and group B prevalence numbers, mapping along the same four groups as in (A) specific cancer type breakdowns within groups are shown. (C) Stage at diagnosis breakdown of the $\mathrm{TbnC}$ and Group B prevalence numbers, mapping along the same four groups as in (A) specific cancer stage at diagnosis breakdowns within groups are shown. 1-lung, trachea and bronchus, 2-secondary malignant neoplasms, 3-non-Hodgkin's lymphoma and non-follicular lymphoma. EoL,end of life;TbnC, treatable but not curable. (CLL - Chronic lymphocytic leukemia, CML - Chronic myeloid leukaemia)

meeting a search criterion (figure 4B). By contrast, TbnC prostate cancer is often defined as TbnC within a month of its diagnosis $(81 \%)$.

\section{Frequency of occurrence of TbnC indicative events and their interrelation}

The occurrence numbers reveal over 191057 occasions of TbnC designating events between 2012 and 2015, among the prevalence cohort of 110615 people (figure 5A). In the occurrence, count criteria 1 (cancer and stage at diagnosis combination) accounts for $35 \%$ of events compared with over $60 \%$ for the prevalence counts (defined by just the first criteria met per person), showing how people who meet criteria 1 at diagnosis often go on to meet multiple other criteria. Out of 67599 people categorised as TbnC based on meeting criteria 1,38\% met at least one additional criterion, if people at EoL are also considered this rises to $50 \%$.
Of the 110615 TbnC cases, $37 \%$ met two or more different criteria (figure 5B,C). For TbnC-EoL cases, the rate of meeting multiple criteria is higher $(65 \%)$ (figure 5C). Across the TbnC and EoL groups, greater number of criteria were met by people in their last year of life (median 2) than those who were not at EoL (TbnC) (median 1); a statistically significant difference (95\% CI 0.37 to $0.38, \mathrm{p}<0.0005)$. In group $\mathrm{B}$, the number meeting multiple criteria is much lower. This may be by definition as group $\mathrm{B}$ can only be defined from criteria 1 or criteria 2 events (figure $5 \mathrm{~A}, \mathrm{~B}$ ) and those who met group $\mathrm{B}$ and any other TbnC criteria are then only included in the TbnC population.

The incidence numbers show 135855 people had their earliest TbnC designating event in 2015. This does not include those who may have had a TbnC designating event before 2012, where data quality was not sufficient 
(A)

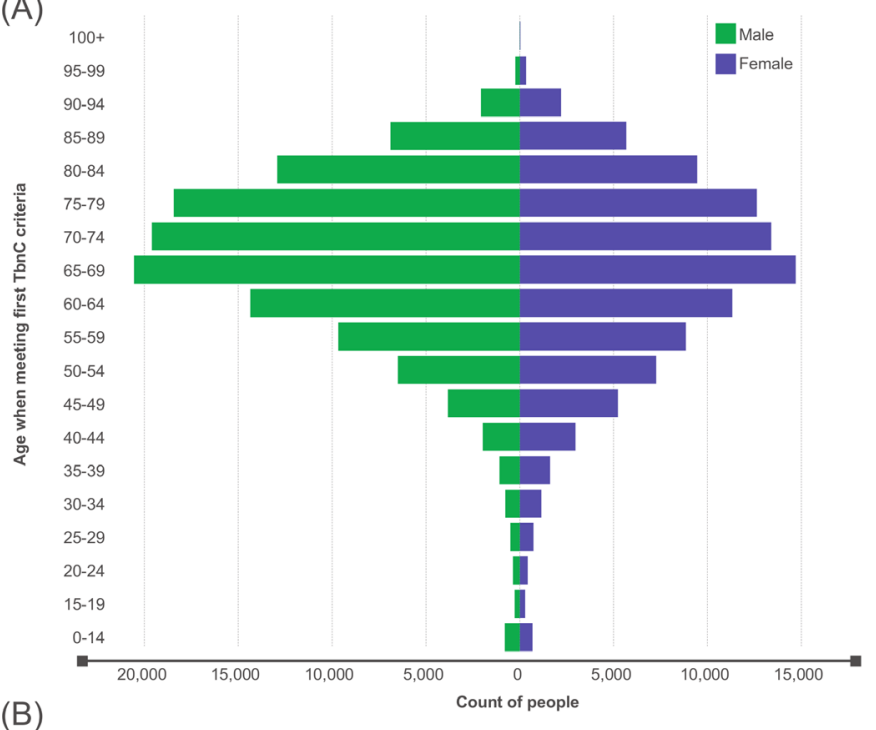

(B)

$$
\begin{aligned}
& 3 \text { month to } 1 \text { month post } \\
& 1 \text { month pre to } 1 \text { year post } \\
& 1 \text { to } 5 \text { years post } \\
& 5 \text { to } 10 \text { years post } \\
& 10+\text { years post Total }
\end{aligned}
$$$$
\text { Total }
$$

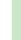

Breast

Brain

Uterus

Melanoma of the skin

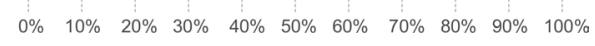

Figure 4 Timing and age in the TbnC cohort. For the $110615 \mathrm{TbnC}$ not EoL cases, each person level record is defined by the earliest criteria met, however multiple criteria may have been met; (A) breakdown of the time from initial diagnosis to meeting of first TbnC criteria for the TbnC not EoL cases. The total for all 11615 cases is show, along with the top five with the largest gap. (B) Population pyramid of the 110616 TbnC not EoL cohort. Age at time of meeting first TbnC criteria. EoL,end of life;TbnC, treatable but not curable.

to apply the algorithm but does include those who died within a year, thus representing an estimated incidence of TbnC cancer in 2015 (figure 5D). Of the 135000 new cases, more than one in two people met the search criteria at the time of cancer diagnosis, while $24782(18 \%)$ of these cases were more than a year out from their cancer diagnosis. Lung cancer was by far the biggest group with 28921 (21\%) new 2015 cases in total. 18431 of these cases were categorised as TbnC based on first meeting criteria 1 -cancer and stage at diagnosis combination, that is, stage 4 lung cancer.

\section{DISCUSSION}

\section{Principal findings}

We have shown, for the first time, that there are over a hundred thousand people in England living with TbnC cancer-cancer that can very rarely be cured but can be treated to help manage their symptoms or slow the progression of the disease and extend their life. We identified 110615 people alive in 2015 in England who met the search criteria for TbnC between 2012 and 2015. There were an additional 51946 people identified who met the search criteria for TbnC but were in their last year of life.

TbnC status is often first defined from stage at diagnosis as this is likely to be the earliest event, but many of these cases are later validated by subsequent TbnC defining events. EoL cases tend to meet even more criteria, potentially indicating the steady accrual of TbnC events as patients transition from treatable disease to their last year of life. When people do not meet the first criterion but do meet others, we hypothesise that this is often due to apparently successful treatment of early stage cancer and then, potentially years later, the development of metastatic disease.

\section{Relation to other studies}

We are here offering a new definition, one that we hope more transparently and usefully defines this cohort, because of that novelty, direct comparison to other related studies is difficult. Nonetheless, as far back as 1996, the idea of managing advanced cancer was being raised to compete with the paradigm of cured versus terminal. ${ }^{17}$ Repeated efforts have been made to quantify and discuss this group. 671819

Previous work described the Three Cancer Groups framework, it included an 'intermediate' survival group, which consists of cancer types where the 1-year survival rate is over $50 \%$ and the 5 -year survival is under $80 \%{ }^{7}$ The intermediate group was designed to explore a similar concept to TbnC and includes some of the same key TbnC and group B cancers selected in criteria 1 such as stage 4 breast and prostate cancer. However, the TbnC algorithm is designed to be more detailed, for example, in splitting out the bladder cancers by stage. The Three Cancer Groups framework aims to generalise the pathways of people living with cancer based on their cancer type and stage at diagnosis, by contrast the TbnC algorithm is designed to use a wide range of indicators to follow individual people over time and classify based on the persons current status in 2015. The Three Cancer Groups framework grouped 'shorter term survival' cancer pathways together but with the additional data in the algorithm, it is possible to separate out those who are TbnC or group B rather than EoL in 2015.

In the USA, at least 300000 people were estimated to be living with advanced cancer. ${ }^{18}$ These represent less than $5 \%$ of the 11.7 million living persons to ever be diagnosed with cancer in $2007 .^{20}$ By contrast, in this study, we identified 162561 people living with TbnC or at EoL in 2015, out of the estimated 2 million living with cancer in the same year, ${ }^{21}$ representing $8 \%$ of the total cancer population in England. A more recent US study estimated the prevalence of metastatic breast cancer alone to be of over 138000 cases, in $2013 .{ }^{19}$ These studies quantify populations related to TbnC but that include different people 

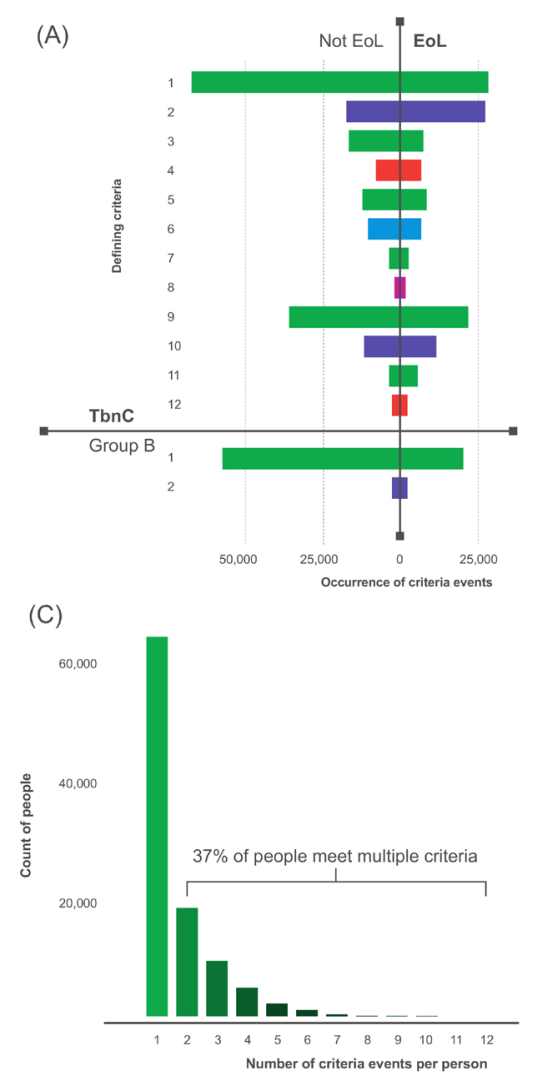

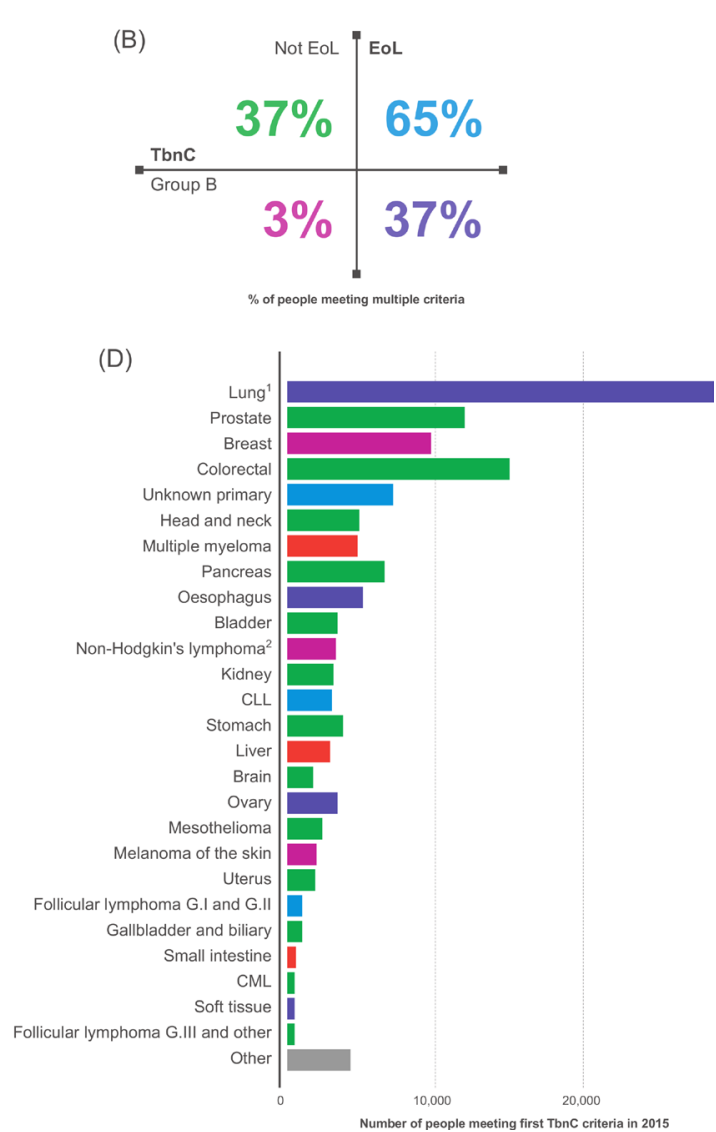

(B)

(D)

Figure 5 The relationships between the criteria. For the 110615 TbnC not EoL cases, each person level record is defined by the earliest criteria met, however, multiple criteria may have been met; $(A)$ event level records showing the occurrence of each criterion across the four groups - note: people are counted multiple times if meeting multiple criteria. (B) Percentage of the groups meeting two or more criteria; 'TbnC-not EoL' group (upper left) and the other three groups as in Fig 2a. (C) Breakdown of number of criteria met by each person in the 'TbnC-not EoL' group. (D) Cancer type breakdown of the TbnC 2015 incidence numbers - note: people are counted multiple times if meeting multiple criteria (group B not included). 1-lung, trachea and bronchus, 2-non-Hodgkin's lymphoma and non-follicular lymphoma. (CLL - Chronic lymphocytic leukemia, CML - Chronic myeloid leukaemia)

and they also use different methods, so the difficulty in comparing these studies has not escaped us. We offer our conceptual and algorithmic definition in the hopes to influence the understanding and terminology around this group, so that more direct comparisons might be possible in the future.

\section{Explanations and implications for clinicians and policymakers} People with TbnC cancer may need tailored emotional, physical and financial support. They may be more likely to undergo multiple rounds of treatments, facing years of scans and follow-ups. This requires enough staff with the right skills and resources to give people with TbnC cancer the personalised care they need and access to appropriate palliative and supportive therapies. People living with TbnC cancer exist in considerable numbers in England, and tailored consideration should be given to their care. Additionally, establishing the concept of TbnC should help healthcare professionals to have a bettershared understanding of prognosis with their patients. This could, in turn, support shared decision-making of treatment and other care options.
Recurrence, particularly distant recurrence, has been poorly recorded in the current PHE data sets. The treatment data sets have only reached a high level of completeness in recent years and there are still some types of activities not well recorded, which has limited the ability to detect all cases of TbnC cancer in our analysis. Additionally, an increased understanding and a clearer definition of recurrence would likely help the process of TbnC case identification. This work demonstrates the importance of improved data collection, both in terms of completeness and the items covered. For example, further development of the Diagnostic Imaging Dataset and primary care data could, in time, contributes significantly to the TbnC discussion.

\section{Strengths and weaknesses}

We carried out this work through a charity-governmental analytical partnership, with in-depth and repeated input from healthcare providers to help build an algorithm and definitions for the population. We also consulted with people living with cancer to help guide our terminology. Additionally, the calculation of these estimates was made 
possible due to the uniquely rich amount of routine health data collected in England, as our strategy relied on the ability to draw information from multiple data sets to build confidence around our work.

There are instances that might be considered TbnC that are not being captured by our criteria as routinely collected health data have its gaps, there is variation in how data are recorded and variation in how fields like those indicating intent might be interpreted. This could impact the number of people who are identified as TbnC in marginal cases. We sought to address some of these concerns by using multiple overlapping criteria, across several datasets, and through seeking repeated clinical input on their development and refinement. We have put weight in the adage 'Hard cases make bad law' and aimed to identify a cohort we had confidence in, but which may miss rare cases of TbnC cancer.

Defining EoL as the 'last year of life' could be argued, however, it is not practical to attempt case by case decisions. Therefore, we adopted an index date previously used in similar work. We believe that some EoL cases would in fact fit the TbnC definition, but others, without options to extend life, can no longer be described as treatable.

This method to identify the TbnC population is likely to require future updating, as some of our definitions are based on specific lists of treatments whose usage and purpose may change. Other changes in clinical practice or database structures may also affect the ability to apply the methods in the future.

The search criteria are not able to cover all circumstances; they would not allow reliable identification of people living with TbnC cancer in a clinical setting. This is not the intent of the study, which is a populationlevel analysis of the group. However, early identification in a clinical setting is essential for access to appropriate specialist care and to allow and encourage meaningful conversations about treatments and plans for the future. ${ }^{5}$

\section{Unanswered questions and future research}

People living with TbnC cancer may face multiple rounds of treatment and it should now be workable, with the developed method, to identify this group and research their treatment profiles and service use within the wealth of data sets from NHS and PHE. It is also possible to investigate the co-morbidity profile of this group, comparing it to that of others living with cancer, which could inform topics such as how the non-cancer health and care workforce may need to interact with this population and the complexity of supporting people with multiple conditions, in the future.

In the search criteria, we were not able to consider the anomalies in cancer staging. For example, we included all stage 4 prostate cancer cases, despite this sometimes only indicating spread to nearby lymph nodes. We would like to see a more consistent staging criterion across all cancer types, as staging methodologies are reviewed.

\section{CONCLUSION}

This study quantifies, for the first time, a significant and under-recognised sub-population of those living with cancer in England who are likely to have increased support needs. We estimate that 110615 people were living with TbnC cancer in England at the end of 2015, with an additional 51946 who had entered their last year of life.

Every person living with cancer faces a different experience, treatments and support should be tailored to these differences. We believe that personalised care is crucial to prolonging and adding quality to the lives of people with cancer. To achieve that aim, however, we must quantify and acknowledge the differences in these experiences of cancer. We report a quantification of people living with TbnC cancer in England, believing this definition of a cancer sub-population to be helpful for planners and health professionals to better consider this group, enabling the formulation of personalised care for them.

Correction notice This article has been corrected since it first published. The provenance and peer review statement has been included.

Twitter Jane Maher @Maherjane

Acknowledgements We thank Macmillan Cancer Support, the National Disease Registration Service, Public Health England, and the wide range of clinicians and experts who gave their advice. Thanks also to colleagues within Macmillan Cancer Support and the National Registration Service who provided review of the manuscript draft. This work uses data that have been provided by patients and collected by the NHS as part of their care and support. The data are collated, maintained and quality assured by the National Registration Service, which is part of Public Health England (PHE).

Contributors All authors contributed to the development and design of the study. FS, JT and JP contributed to the coding required to extract the numbers from the datasets, performed the calculations and produced the accompanying protocol and outputs. RW, JM and FS designed the analysis approach, analysed and interpreted the results and formulated the conclusions. AM contributed clinical expertise, LH contributed data expertise and GF facilitated stakeholder engagement. RW and FS wrote the manuscript. RW and FS contributed equally to this paper. All authors contributed to the manuscript and all authors read and approved the final manuscript.

Funding The authors have not declared a specific grant for this research from any funding agency in the public, commercial or not-for-profit sectors.

Competing interests None declared.

Patient consent for publication Not required.

Provenance and peer review Not commissioned; externally peer reviewed.

Data availability statement Data are available via a public, open access release. Detailed methods and the resulting de-identified datasets are publicly available at: http://www.ncin.org.uk/view?rid=4153.

Supplemental material This content has been supplied by the author(s). It has not been vetted by BMJ Publishing Group Limited (BMJ) and may not have been peer-reviewed. Any opinions or recommendations discussed are solely those of the author(s) and are not endorsed by BMJ. BMJ disclaims all liability and responsibility arising from any reliance placed on the content. Where the content includes any translated material, BMJ does not warrant the accuracy and reliability of the translations (including but not limited to local regulations, clinical guidelines, terminology, drug names and drug dosages), and is not responsible for any error and/or omissions arising from translation and adaptation or otherwise.

Open access This is an open access article distributed in accordance with the Creative Commons Attribution Non Commercial (CC BY-NC 4.0) license, which permits others to distribute, remix, adapt, build upon this work non-commercially, and license their derivative works on different terms, provided the original work is properly cited, appropriate credit is given, any changes made indicated, and the use is non-commercial. See: http://creativecommons.org/licenses/by-nc/4.0/. 
ORCID iD

Fintan Stanley http://orcid.org/0000-0002-6740-3280

\section{REFERENCES}

1 Maher J, Velikova G, Betteley A. Incurable, but treatable: how to address challenges for an emerging group. BMJ Support Palliat Care 2015;5:322-4.

2 Molassiotis A, Wilson B, Blair S, et al. Living with multiple myeloma: experiences of patients and their informal caregivers. Support Care Cancer 2011;19:101-11.

3 Mosher CE, Adams RN, Helft PR, et al. Family caregiving challenges in advanced colorectal cancer: patient and caregiver perspectives. Support Care Cancer 2016;24:2017-24.

4 Carlson M. Living scan to scan. Cure, 2018.

5 Shilling V, Starkings R, Jenkins V, et al. The pervasive nature of uncertainty-a qualitative study of patients with advanced cancer and their informal caregivers. J Cancer Surviv 2017;11:590-603.

6 Yip K, McConnell H, Alonzi R, et al. Using routinely collected data to stratify prostate cancer patients into phases of care in the United Kingdom: implications for resource allocation and the cancer survivorship programme. Br J Cancer 2015;112:1594-602.

7 McConnell H, White R, Maher J. Categorising cancers to enable tailored care planning through a secondary analysis of cancer registration data in the UK. BMJ Open 2017;7:e016797.

8 Doorenbos AZ, Given CW, Given B, et al. Symptom experience in the last year of life among individuals with cancer. J Pain Symptom Manage 2006;32:403-12.

9 Bennett MI, Ziegler L, Allsop M, et al. What determines duration of palliative care before death for patients with advanced disease? A retrospective cohort study of community and hospital palliative care provision in a large UK City. BMJ Open 2016;6:e012576.

10 Public Health England. Phe cancer data sets, linkage and availability (v1.1), 2017: 1-10.

11 England, P. H. a guide to NCRAS data and its availability about public health England, 2020. Available: https://www.google.com/ url?sa=t\&rct=j\&q=\&esrc=s\&source=web\&cd=\&ved=2ahUKEwju98e vhYzsAhXWQRUIHVCODIQQFjABegQIARAB\&url=https://assets. publishing.service.gov.uk/government/uploads/system/uploads/ attachment data/file/884776/A guide to NC

12 von Elm E, Altman DG, Egger M, et al. The strengthening the reporting of observational studies in epidemiology (STROBE) statement: guidelines for reporting observational studies. Bull World Health Organ 2007;85:867-72.

13 Parliament of the United Kingdom. National health service act, 2006

14 The UK Caldicott Guardian Council. A manual for Caldicott guardians. A manual for Caldicott guardians, 2017.

15 Parliament of the United Kingdom. Data protection act. Parliament of the United Kingdom, 2018.

16 European Parliament and Council of European Union. General data protection regulation, 2016.

17 Rennie J, Rusting R. Making headway against cancer. Sci Am 1996;275:56-9

18 Haylock PJ. Advanced cancer: emergence of a new survivor population. Semin Oncol Nurs 2010;26:144-50.

19 Mariotto AB, Etzioni R, Hurlbert M, et al. Estimation of the number of women living with metastatic breast cancer in the United States. Cancer Epidemiol Biomarkers Prev 2017;26:809-15.

20 Cancer Survivors-United states, 2007. JAMA 2011;305:2281-2.

21 Transforming Cancer Services Team,, Macmillan Cancer Support \& National Disease Registration Service. Cancer Prevalence in England - 21 year prevalence by demographic measures at Local Authority, 2018. Available: http://www.ncin.org.uk/view?rid=3635 